\title{
SETS DEFINABLE OVER FINITE FIELDS: THEIR ZETA-FUNCTIONS
}

\author{
BY \\ CATARINA KIEFE(1)
}

\begin{abstract}
Sets definable over finite fields are introduced. The rationality of the logarithmic derivative of their zeta-function is established, an application of purely algebraic content is given. The ingredients used are a result of Dwork on algebraic varieties over finite fields and model-theoretic tools.
\end{abstract}

1. Introduction. In [6] Dwork proved the rationality of the zeta-function of a variety over a finite field. The main result of this paper is to extend this as far as possible to sets definable over finite fields. In this case, the zeta-function need no longer be rational, as illustrated by the set defined over the finite field with $p$ elements ( $p$ odd prime) by the formula

$$
\exists x\left(x^{2}-y=0\right) \text {. }
$$

However, the logarithmic derivative of the zeta-function, i.e., the Poincaré series, turns out always rational.

The result is found using model-theoretic tools: an extension by definitions of the theory of finite fields in ordinary field language in given: this extension is shown to admit elimination of quantifiers (by virtue of a generalization of the Shoenfield Quantifier Elimination Theorem [8]), this yields a characterization of sets definable over finite fields, and the Poincaré series for these can now be proved to be rational by some computations; although the zeta-function need not be rational, from the computation one can conclude that it can always be expressed as the radical of a rational function.

Unexplained notation follows Shoenfield [7] and Bell and Slomson [4].

2. A semantic characterization of elimination of quantifiers. Let $\tau$ be a similarity type, $L_{\tau}$ the first-order language of type $\tau$; let $\Lambda$ be a theory in language $L_{\tau}$.

Received by the editors

AMS (MOS) subject classifications (1970). Primary 02H15, $12 \mathrm{C} 99,12 \mathrm{~L} 99$.

Key words and phrases. Finite and pseudo-finite fields, varieties, definable sets, zetafunction, elimination of quantifiers.

(1) The results presented in this paper are part of the author's doctoral dissertation, written at the State University of New York at Stony Brook, under the supervision of James Ax; the author wishes to thank Professor Ax for encouragement and advice. 
Definition 1. We say that $\Lambda$ satisfies the isomorphism condition if for every two models $A$ and $A^{\prime}$ of $\Lambda$ and every isomorphism $\theta$ of substructures of $A$ and $A^{\prime}$, there is an extension of $\theta$ which is an isomorphism of a submodel of $A$ and a submodel of $A^{\prime}$.

DEFINITION 2. We say that $\Lambda$ satisfies the submodel condition if for every model $B$ of $\Lambda$, every submodel $A$ of $B$, and every closed simply existential formula $\varphi$ of $L_{\tau, A}$, we have

$$
A \vDash \varphi \Longleftrightarrow B \vDash \varphi \text {. }
$$

The following theorem is well known $[8$, p. 85]:

QuANTIFIER Elimination THEOREM. If $\Lambda$ satisfies the isomorphism condition and the submodel condition, then $\Lambda$ admits elimination of quantifiers.

The Quantifier Elimination Theorem gives a sufficient condition for a theory to admit elimination of quantifiers. However, this condition is not necessary, as is established by the following counterexample, due to Allan Adler.

Counterexample. Let $\Gamma$ denote the "theory of independent events", described as follows:

LANGUAGE OF $\Gamma$ : no constant symbols no function symbols

a countable set $\left\{\rho_{n} \mid n \in \omega\right\}$ of unary predicate symbols.

Axioms of $\Gamma$ : for every ordered pair $(S, T)$ of finite subsets of $\omega$ such that $S \cap T$ is empty we have an axiom

$$
\left.A_{(S, T)}:(\exists x)\left(\bigwedge_{n \in S} \rho_{n}(x) \wedge \bigwedge_{n \in T}\right\urcorner \rho_{n}(x)\right) \text {. }
$$

$\Gamma$ admits elimination of quantifiers as can be proved by applying Lemma 3 in $[8$, p. 83]. To establish the counterexample one shows that $\Gamma$ does not satisfy the isomorphism condition: indeed, we define two subsets $M, N$ of $[0,1]$ as follows:

First, we define sequences $\left\{M_{n}\right\}_{n \in \omega},\left\{N_{n}\right\}_{n \in \omega}$ by $M_{0}=N_{0}=\{0\}$, if $M_{0}, \ldots, M_{n}, N_{0}, \ldots, N_{n}$ are known, choose $\xi_{1}, \ldots, \xi_{2^{n+1}}, \eta_{1}, \ldots, \eta_{2 n+1}$ in $[0,1]$ such that all are irrational,

$$
\xi_{j}, \eta_{j} \in\left[(j-1) / 2^{n+1}, j / 2^{n+1}\right] \quad\left(j=1, \ldots, 2^{n+1}\right),
$$

all are distinct, and none are contained in $M_{n}$ or $N_{n}$. We put $M_{n+1}=M_{n} \cup$ $\left\{\xi_{1}, \ldots, \xi_{2^{n+1}}\right\}, N_{n+1}=N_{n} \cup\left\{\eta_{1}, \ldots, \eta_{2^{n+1}}\right\}$.

We now define $M=\bigcup_{n \in \omega} M_{n}, N=\bigcup_{n \in \omega} N_{n}$.

We make $M, N$ models of $\Gamma$ by interpreting $\rho_{n}(x)$ to mean that the $n$th 
binary digit of $x$ is 1 . The axioms then simply require that $M$ and $N$ should each have nonempty intersection with each dyadic interval $\left[j / 2^{n},(j+1) / 2^{n}\right]$, and are satisfied by construction.

$M_{0}=N_{0}=\{0\}$ are isomorphic substructures of $M$ and $N$. However, any isomorphism of submodels of $M$ and $N$ must take an irrational number into itself. Since $M \cap N=\{0\}$, the isomorphism condition fails.

The Quantifier Elimination Theorem is now going to be extended to a necessary and sufficient condition, therewith yielding a semantic characterization of the elimination of quantifiers. We need

DEFINITION 3. We say that $\Lambda$ satisfies the weak isomorphism condition if for every two models $A$ and $A^{\prime}$ of $\Lambda$ and every isomorphism $\theta$ of a substructure of $A$ and a substructure of $A^{\prime}$, there is an elementary extension $A^{\prime \prime}$ of $A^{\prime}$ and an extension of $\theta$ which is an isomorphism of a submodel of $A$ and a submodel of $A^{\prime \prime}$.

We then have

THEOREM 1. $\Lambda$ admits elimination of quantifiers if and only if $\Lambda$ is model-complete and $\Lambda$ satisfies the weak isomorphism condition. $\left({ }^{2}\right)$

Proof. $\Leftarrow:$ The techniques used in [8] to prove the Quantifier Elimination Theorem can easily be adapted to prove that quantifiers can be eliminated even with these weaker hypotheses. (2)

$\Rightarrow$ : Model-completeness follows trivially.

3. A language in which the theory of finite fields admits elimination of quantifiers. We now describe a language and theory of finite fields in this language which admits elimination of quantifiers:

LANGUAGE: function symbols: + (addition)

$$
\text { - (multiplication) }
$$

$$
\begin{array}{ll} 
& - \text { (subtraction) } \\
\text { constant symbols: } & 1 \text { (unity) } \\
& 0 \text { (additive identity) }
\end{array}
$$$$
\text { predicate symbols: }=\text { (equality). }
$$

This language is the ordinary field language; henceforth, we denote it $L_{\tau}$. Now, we introduce for every positive integer $n$ an $n+1$-ary predicate symbol: $\varphi_{n} . L_{\tau^{\prime}}$ denotes the language obtained by adjoining the predicate symbols $\left\{\varphi_{n} \mid n \in Z_{>0}\right\}$ to $L_{\tau}$.

(2) Conversely, the necessity of these hypotheses follows easily by, e.g., an application of Frayne's Lemma [4, p. 161].

It has been brought to my attention that Theorem 13.1 of $[7$, p. 63] yields a characterization of elimination of quantifiers very close to this one. However, the one presented here appears to be somewhat more convenient for the purpose of this paper. 
We now denote

$\Sigma$-the theory of finite fields in $L_{\tau}$ (i.e., the set of sentences of $L_{\tau}$ satisfied by all finite fields)

$\pi$-the theory of pseudo-finite fields in $L_{\tau}$ (i.e., the set of sentences of $L_{\tau}$ satisfied by all the infinite models of $\Sigma$ ).

In $[2$, p. 255, Theorem 5], a recursive axiomatization for $\pi$ can be found.

Naturally, $\Sigma \subseteq \pi$, i.e., $F \vDash \pi \Rightarrow F \vDash \Sigma$.

Now, we let $\pi^{\prime}$ and $\Sigma^{\prime}$ be the theories in the language $L_{\tau^{\prime}}$ obtained by taking for axioms respectively

$\pi \cup\left\{\forall x_{0} \cdots \forall x_{n}\left(\varphi_{n}\left(x_{0}, \ldots, x_{n}\right) \leftrightarrow \exists y\left(x_{n} y^{n}+\cdots+x_{0}=0\right)\right) \mid n \in Z_{>0}\right\}$

and

$$
\begin{aligned}
\Sigma \cup\left\{\forall x_{0} \cdots \forall x_{0}\right. & \left(\left(\neg \exists y_{1} \cdots \exists y_{n}\left(\bigwedge_{\substack{i, j=1 \\
i \neq j}}^{n} y_{i} \neq y_{j} \wedge \forall y\left(\bigvee_{i=1}^{n} y=y_{i}\right)\right)\right.\right. \\
& \left.\rightarrow\left(\varphi_{n}\left(x_{0}, \ldots, x_{n}\right) \leftrightarrow \exists y\left(x_{n} y^{n}+\cdots+x_{0}=0\right)\right)\right) \\
& \wedge\left(\exists y_{1} \cdots \exists y_{n}\left(\bigwedge_{\substack{i, j=1 \\
i \neq j}}^{n} y_{i} \neq y_{j} \wedge \forall y\left(\bigvee_{i=1}^{n} y=y_{i}\right)\right)\right. \\
& \left.\left.\left.\rightarrow\left(\varphi_{n}\left(x_{0}, \ldots, x_{n}\right) \leftrightarrow \forall y\left(y=0 \vee \bigvee_{i=1}^{n-1} y=x_{0}^{i}\right)\right)\right)\right)\left.\right|_{n \in Z_{>0}}\right\}
\end{aligned}
$$

REMARKS. (a) $\Sigma^{\prime}$ is an extension by definitions of $\Sigma$; given $F=\Sigma, F$ becomes a model of $\Sigma^{\prime}$ in a canonical way:

Case 1. $F$ is infinite-then we define the $n+1$-ary relation $\varphi_{n}^{F}$ by

$$
\left(a_{0}, \ldots, a_{n}\right) \in \varphi_{n}^{F} \Longleftrightarrow \text { the polynomial } a_{n} y^{n}+\cdots+a_{0} \text { has a root in } F \text {. }
$$

Case 2. $F$ is finite with $k$ elements-then $\varphi_{n}^{F}$ is defined as before if $n \neq k$, and $\varphi_{k}^{F}$ is defined by $\left(a_{0}, \ldots, a_{k}\right) \in \varphi_{k}^{F} \Longleftrightarrow a_{0}$ is a generator of $F^{*}$ (multiplicative subgroup of $F$ ).

(b) $F=\pi^{\prime} \Longleftrightarrow F_{I}=\Sigma^{\prime}$ and $F$ is infinite,

(c) $F \vDash \Sigma^{\prime} \Rightarrow\left(F\right.$ finite with $k$ elements $\left.\Longleftrightarrow(0,0, \ldots, 0,1) \notin \varphi_{k}^{F}\right)$.

LEMMA 1. $\pi^{\prime}$ admits elimination of quantifiers $\Longleftrightarrow \Sigma^{\prime}$ admits elimination of quantifiers. 
Proof. $\Leftarrow$ : obvious, since $\Sigma^{\prime} \subset \pi^{\prime}$.

$\Rightarrow$ : by Theorem 1 , it suffices to show that

(i) $\pi^{\prime}$ model-complete $\Rightarrow \Sigma^{\prime}$ model-complete, and

(ii) $\pi^{\prime}$ satisfies weak isomorphism condition $\Rightarrow \Sigma^{\prime}$ satisfies weak isomorphism condition.

(i) Let $F_{j}=\Sigma^{\prime}(j=1,2)$ and $F_{1} \subseteq F_{2}$.

If $F_{1}$ is infinite, $F_{j}=\pi^{\prime}(j=1,2)$ and $F_{1} \leqslant F_{2}$ follows from hypothesis.

If $F_{1}$ is finite with $k$ elements,

$(1,0, \ldots, 0,1) \notin \varphi_{k}^{F_{1}}=\varphi_{k}^{F_{2}} \cap F_{1}^{k}$

$$
\Rightarrow(1,0, \ldots, 0,1) \notin \varphi_{k}^{F_{2}} \Rightarrow F_{2} \text { finite } k \text { elements } \Rightarrow F_{1}=F_{2} .
$$

(ii) Let $F_{j}=\Sigma^{\prime}(j=1,2)$ and $\theta$ an isomorphism of nonempty-substructures: esis.

If both $F_{1}$ and $F_{2}$ are infinite, $F_{j}=\pi^{\prime}$, and $\theta$ can be extended by hypoth-

If $F_{1}$ is finite with $k$ elements, $(1,0, \ldots, 0,1) \notin \varphi_{k}^{F_{1}} \Rightarrow(1, \ldots, 0,1) \notin$ $\varphi_{k}^{F}$ (because $\theta$ is an isomorphism) $\Rightarrow F_{2}$ is finite with $k$ elements. Hence $\theta$ is an isomorphism of two subrings of two fields with $k$ elements, the subrings containing the prime fields; so, obviously, $\theta$ can be extended to the fields with $k$ elements.

If $F_{2}$ is finite with $k$ elements a similar reasoning holds.

THEOREM 2. $\pi^{\prime}$ admits elimination of quantifiers.

Proof. By Theorem 1, this proof is immediately reduced to the proof of the following two lemmas:

LEMMA 2. $\pi^{\prime}$ is model-complete.

LEMMA 3. $\pi$ ' satisfies the weak isomorphism condition.

For the proofs of Lemmas 2 and 3 we need

Lemma 4. Let $F_{i}=\pi^{\prime}(i=1,2)$, and assume that $F_{1}$ is a subfield of $F_{2}$; then $F_{1} \subseteq F_{2}$ (i.e., for all $\left.n \in Z_{>0}, \varphi_{n}^{F_{1}}=\varphi_{n}^{F_{2}} \cap F_{1}^{n+1}\right) \Longleftrightarrow F_{1}$ is relatively algebraically closed in $F_{2}$.

We also use

LEMMA 5. Let $\Lambda$ be a theory without finite models in a language of cardinality $\aleph_{0}$. Then: $\Lambda$ model-complete $\Longleftrightarrow$ for any model $A \vDash \Lambda$ of cardinality $N_{0}$,

$\Lambda \cup$ Diagram of $A$ is complete. 
Proof. $\Rightarrow$ : obvious, from one of the current definitions of model-completeness.

$\Leftarrow$ : let $B_{1}, B_{2}=\Lambda, B_{1} \subseteq B_{2}$.

By Robinson's test for model-completeness; it suffices to show that if $\varphi$ is a primitive sentence in the language of $B_{1}$ and $B_{2} \vDash \varphi$, then $B_{1} \vDash \varphi$. Indeed: in $\varphi$ occur only a finite set $S$ of contants designating elements of $\left|B_{1}\right|$. By Skolem-Loewenheim, we can extend $S$ to a model $B_{3} \mid=\Lambda$ such that $S \subseteq\left|B_{3}\right|$ and $B_{3} \leqslant B_{1} \subseteq B_{2}$ and card $\left|B_{3}\right|=\aleph_{0}$. By hypothesis, Diag $B_{3} \cup \Lambda$ is complete. But

$$
\begin{aligned}
& B_{2} \vDash \operatorname{Diag} B_{3} \cup \Lambda, \text { and } \\
& B_{2}=\varphi \text {, so } \\
& \text { Diag } B_{3} \cup \Lambda \vDash \varphi \text {, hence } B_{3} \vDash \varphi \\
& \text { and } B_{3} \leqslant B_{1} \Rightarrow B_{1} \vDash \varphi \text {. Q.E.D. }
\end{aligned}
$$

Proof of Lemma 2. Since $\pi$ ' has no finite models, by Lemma 5, to prove that $\pi^{\prime}$ is model-complete it suffices to show that $F=\pi^{\prime}$ and card $F=\aleph_{0} \Rightarrow$ $\pi^{\prime} \cup \operatorname{Diag} F$ complete: Let $F_{1}, F_{2}=\pi^{\prime} \cup \operatorname{Diag} F$; we want to show that

$$
F_{1} \equiv F_{2} \quad \text { (in language } L_{\tau^{\prime \prime}} \text { of } \pi^{\prime} \cup \operatorname{Diag} F \text { ). }
$$

We may assume that $F \subseteq F_{i}(i=1,2)$, and by Loewenheim-Skolem, we may assume card $F_{i}=\aleph_{0} \quad(i=1,2)$.

Now let $D$ be a nonprincipal ultrafilter on the set of positive integers $I$; let

$$
\epsilon_{i}=F_{i}^{I} / D \quad(i=1,2),
$$

since $\epsilon_{i}$ is pseudo-finite, $\epsilon_{i}$ is hyper-finite; (cf. definition in [2, p. 246]) so we have $F \subseteq F_{i} \leqslant \epsilon_{i}$, with $\epsilon_{i}$ hyper-finite; by Lemma $4, F$ is relatively algebraically closed in $\epsilon_{i}(i=1,2)$; and also card $\epsilon_{1}=$ card $\epsilon_{2}>$ card $F$. Hence, by [2, p. 247, Theorem 1], $\epsilon_{1}$ and $\epsilon_{2}$ are isomorphic as fields over $F$; but this implies that they are isomorphic as structures of type $\tau^{\prime \prime}$, since the $\varphi_{n}^{\epsilon_{i}}$ relations are "algebraic", i.e., preserved under field-isomorphisms. Hence

$$
\begin{aligned}
& F_{1} \leqslant \epsilon_{1} \simeq \epsilon_{2} \geqslant F_{2}, \text { so } \\
& F_{1} \equiv F_{2} . \quad \text { Q.E.D. }
\end{aligned}
$$

Proof of Lemma 3. Let $\epsilon_{i} \vDash \pi^{\prime}(i=1,2), D_{i} \subseteq \epsilon_{i}$ and $\theta: D_{1} \rightarrow D_{2}$ be an isomorphism (of structures of type $\tau^{\prime}$ ).

$D_{i}$ is a substructure of $\epsilon_{i}$, hence an integral domain. Let $F_{i}$ be the quotient field of $D_{i}: F_{i} \subseteq \epsilon_{i}$, and certainly $\theta$ extends to a field-isomorphism $\theta: F_{1} \rightarrow F_{2}$. $\theta$ is also an isomorphism of structures of type $\tau^{\prime}$, as can be easily checked; so $\theta$ 
has the following property:

$$
\begin{aligned}
& a_{n} x^{n}+\cdots+a_{0} \in F_{1}[x] \text { has a zero in } \epsilon_{1} \\
& \Longleftrightarrow \theta\left(a_{n}\right) x^{n}+\cdots+\theta\left(a_{0}\right) \in F_{2}[x] \text { has a zero in } \epsilon_{2} .
\end{aligned}
$$

Now let $\tilde{F}_{i}^{r}$ be the relative algebraic closure of $F_{i}$ in $\epsilon_{i}$. Of course, we again have that

$$
\begin{aligned}
& a_{n} x^{n}+\cdots+a_{0} \in F_{1}[x] \text { has a zero in } \tilde{F}_{1}^{r} \\
& \Longleftrightarrow \theta\left(a_{n}\right) x^{n}+\cdots+\theta\left(a_{0}\right) \in F_{2}[x] \text { has a zero in } \tilde{F}_{2}^{r} .
\end{aligned}
$$

Hence by $[1$, p. 172 , Lemma 5], we can extend $\theta$ to a field-isomorphism $\theta$ : $\tilde{F}_{1}^{r} \rightarrow \widetilde{F}_{2}^{r} . \theta$ is still an isomorphism of structures of type $\tau^{\prime}$ because now

$$
\begin{aligned}
& \left(a_{0}, \ldots, a_{n}\right) \in \varphi_{n} \tilde{F}_{1}^{r}=\varphi_{n}^{\epsilon} \cap \tilde{F}_{1}^{r+1} \Longleftrightarrow a_{n} x^{n}+\cdots+a_{0} \\
& \text { has a zero in } \epsilon_{1} \Longleftrightarrow a_{n} x^{n}+\cdots+a_{0} \text { has a zero in } \tilde{F}_{1}^{r} \\
& \Leftrightarrow \theta\left(a_{n}\right) x^{n}+\cdots+\theta\left(a_{0}\right) \text { has a zero in } \tilde{F}_{2}^{r} \\
& \Leftrightarrow \theta\left(a_{n}\right) x^{n}+\cdots+\theta\left(a_{0}\right) \text { has a zero in } \epsilon_{2} \\
& \Leftrightarrow\left(\theta\left(a_{0}\right), \ldots,\left(a_{n}\right)\right) \in \varphi_{n}^{\epsilon} \cap F_{2}^{r^{n+1}}=\varphi_{n} F_{1}^{r} .
\end{aligned}
$$

Let $\alpha=\operatorname{card} \epsilon_{2}$. By upward Loewenheim-Skolem, let $H_{2}^{\prime}$ be such that $\epsilon_{2} \leqslant H_{2}^{\prime}$ and card $H_{2}^{\prime}=\alpha^{+}$. Now, let $H_{2}$ be such that $\epsilon_{2} \leqslant H_{2}^{\prime} \leqslant H_{2}$, card $H_{2}$ $=2^{\alpha}$ and $H_{2}$ is $\alpha^{+}$-saturated [4, Theorem 11.1.7].

Then we have that $\epsilon_{2} \leqslant H_{2}, H_{2}$ is hyper-finite, card $H_{2}=2^{\alpha}$ and $\tilde{F}_{2}^{r}$ is relatively algebraically closed in $\mathrm{H}_{2}$ (because $\epsilon_{2} \leqslant \mathrm{H}_{2}$ ).

Let $\beta=$ card $\widetilde{F}_{1}^{r}=$ card $\widetilde{F}_{2}^{r} \leqslant \alpha<2^{\alpha}$; by downward Loewenheim-Skolem, let $H_{1}$ be such that $\tilde{F}_{1}^{r} \subseteq H_{1} \leqslant \epsilon_{1}$ and card $H_{1}=\beta$. Then we know that $H_{1}$ is quasi-finite (because $H_{1} \leqslant \epsilon_{1} \Rightarrow H_{1} \vDash \pi^{\prime}$ ), card $H_{1}<$ card $H_{2}$, and $\tilde{F}_{1}^{r}$ is relatively algebraically closed in $H_{1}$. So by [2, Lemma 2] we can extend $\theta$ to a field-monomorphism $\theta: H_{1} \rightarrow H_{2}$ such that $\theta\left(H_{1}\right)$ is relatively algebraically closed in $\mathrm{H}_{2}$.

If we take $\varphi_{n}^{\theta\left(H_{1}\right)}$ to be defined on $\theta\left(H_{1}\right)$ through $\theta$, we get, since $H_{1} \vDash$ $\pi^{\prime}$, that $\theta\left(H_{1}\right) \vDash \pi^{\prime}$. But now $H_{2}, \theta\left(H_{1}\right) \vDash \pi^{\prime}, \theta\left(H_{1}\right)$ is a subfield of $H_{2}$, and is relatively algebraically closed in $H_{2}$. Then Lemma 4 applies to show that $\theta\left(H_{1}\right)$ $\subseteq H_{2}$, i.e., with $\varphi_{n}^{\theta\left(H_{1}\right)}$ defined as above, $\theta\left(H_{1}\right)$ is a submodel of $H_{2}$. Hence we have proved the weak isomorphism condition. Q.E.D.

4. Sets definable over a finite field: the rationality of their Poincaré series. In this section, we shall use the following

Notation. $L_{\tau}$-ordinary field language, as described in $\S 3$.

$L_{\tau^{\prime}}$-ordinary field language with all the $n+1$-ary predicate symbols $\varphi_{n}$ adjoined $\left(n \in Z_{>0}\right)$. 
$\Sigma$-theory of finite fields in $L_{\tau}$.

$\Sigma^{\prime}$-theory of finite fields with defining axioms for $\varphi_{n}$ adjoined (as in $\S 3$ ). $k$-finite field of cardinality $q$.

$L_{\tau, k}-L_{\tau}$ with $q$ new constant symbol adjoined.

$k_{s}$-unique extension of $k$ of degree $s$.

$\widetilde{k}$-algebraic closure of $k$.

Definition 4. Let $U=\left\{U_{s}\right\}_{s \in Z_{>0}}$ with $U_{s} \subset k_{s}^{r}, \forall s \in Z_{>0}$; then $U$ is called a definable $r$-set over $k \Leftrightarrow$ there exists a formula $\varphi$ in $L_{\tau, k}$ with $r$ free variables such that

$$
U_{s}=\left\{\left(a_{1}, \ldots, a_{r}\right) \in k_{s}^{r} \mid k_{s}=\varphi\left[a_{1}, \ldots, a_{r}\right]\right\}, \quad \forall s \in \mathbf{Z}_{>0} .
$$

We then say that $U$ is defined by $\varphi$.

REMARK. If $U$ is definable over $k$, the formula defining $U$ is not unique: in fact, every formula representing the same element in the $r$ th Lindenbaum algebra of $\Sigma$ will also define $U$.

Definition 5. Say $U$ is a definable $r$-set, defined by $\varphi$. We have $U_{s}=$ $\left\{\left(a_{1}, \ldots, a_{r}\right) \in k_{s}^{r} \mid k_{s}=\varphi\left[a_{1}, \ldots, a_{r}\right]\right\}$; the zeta-function of $U$ is defined to be the formal power series in $t$

$$
\zeta_{U}(t)=\exp \sum_{s=1}^{\infty} \frac{N_{s}(U)}{s} t^{s}
$$

where $N_{s}(U)=\# U_{s}=$ cardinality of $U_{s}$. Following terminology used in [5, p. 47] we let the Poincaré series of $U$ be defined by

$$
\pi_{U}(t)=t \frac{d}{d t} \log \zeta_{U}(t)=\sum_{s=1}^{\infty} N_{s}(U) t^{s}
$$

The main result of this section is

TheOREM 3. The Poincaré series of a definable set is rational.(3)

DEFINITION 6. A definable $r$-set $V$ over $k$ will be called a variety over $k$ if it can be defined by a formula of type

$$
\begin{gathered}
\bigwedge_{i=1}^{n} p_{i}\left(x_{1}, \ldots, x_{r}\right)=0, \text { with } \\
p_{i}\left(x_{1}, \ldots, x_{r}\right) \in k\left[x_{1}, \ldots, x_{r}\right] \quad(i=1, \ldots, n) .
\end{gathered}
$$

DEFinition 7. A definable $r$-set will be called primitive if it can be defined by a formula of type

(3) As usual, a formal power series is called rational when it is the quotient of two polynomials. 


$$
\bigwedge_{i=1}^{n} p_{i}\left(x_{1}, \ldots, x_{r}\right)=0 \wedge \bigwedge_{i=1}^{m} q_{i}\left(x_{1}, \ldots, x_{r}\right) \neq 0
$$

with $p_{i}(\bar{x}), q_{j}(\bar{x}) \in k[\bar{x}],(i=1, \ldots, n ; j=1, \ldots, m)$.

DEFinition 8. A definable set will be called constructible if it can be defined by a formula which is quantifier free in $L_{\tau, k}$.

DEFinition 9. Let $U=\left\{U_{s}\right\}_{s \in Z_{>0}}$ and $V=\left\{V_{s}\right\}_{s \in Z_{>0}}$ be definable $r$-sets. We define the union, intersection and difference of $U$ and $V$ "pointwise", i.e., by

$$
\begin{gathered}
(U \cup V)_{s}=U_{s} \cup V_{s}, \quad(U \cap V)_{s}=U_{s} \cap V_{s}, \\
(U-V)_{s}=U_{s}-V_{s}, \quad \forall s \in Z_{>0} .
\end{gathered}
$$

LEMMA 6. If $U$ is a constructible set, then $\zeta_{U}(t)$ is a rational function. Hence, so is $\pi_{U}(t)$.

Proof. Dwork [6] showed that $\zeta_{V-W}(t)$ is rational, for $V, W$ varieties.

Any primitive set $P_{n}$ is a difference of varieties: in fact, if $P$ is defined by $\bigwedge_{i=1}^{n} p_{i}(\bar{x})=0 \wedge \bigwedge_{j=1}^{m} q_{j}(\bar{x}) \neq 0$, we have that

$$
\Sigma \vdash\left(\bigwedge_{i=1}^{n} p_{i}(\bar{x}) \wedge \bigwedge_{j=1}^{m} q_{j}(\bar{x}) \neq 0\right) \leftrightarrow\left(\bigwedge_{i=1}^{n} p_{i}(x)=0 \wedge \prod_{j=1}^{m} q_{j} \neq 0\right)
$$

So if $V$ is defined by $\bigwedge_{i=1}^{n} p_{i}(\bar{x})=0$ and $W$ is defined by $\left(\prod_{j=1}^{m} q_{j}(\bar{x})\right)=0$, then $P=V-W$. So the Lemma holds for primitive sets.

Now observe that the intersection of primitive sets is primitive; on the other hand, any constructible set is the union of primitive sets, i.e., if $U$ is constructible, there exist primitive sets $P_{1}, \ldots, P_{n}$ such that $U=\bigcup_{i=1}^{n} P_{i}$ and so $U_{s}=$ $\bigcup_{i=1}^{n}\left(P_{i}\right)_{s}$; it is easily verified that

$$
\begin{gathered}
\#\left(\bigcup_{i=1}^{n}\left(P_{i}\right)_{s}\right)=\sum_{\phi \neq B \subseteq\{1, \ldots, n\}}(-1)^{\# B+1} \#\left(\bigcap_{i \in B}\left(P_{i}\right)_{s}\right), \text { i.e., } \\
N_{s}(U)=\sum_{\phi \neq B \subseteq\{1, \ldots, n\}}(-1)^{\# B+1} N_{s}\left(\bigcap_{i \in B} P_{i}\right)=\sum_{\phi \neq B \subseteq\{1, \ldots, n\}}(-1)^{\# B+1} N_{s}\left(P_{B}\right),
\end{gathered}
$$

where $P_{B}=\bigcap_{i \in B} P_{i}$, for all $B \subseteq\{1, \ldots, n\}$. But $P_{B}$ is a primitive set, hence $\zeta_{P_{B}}(t)$ is rational, so

$$
\zeta_{U}(t)=\prod_{\phi \neq B \subseteq\{1, \ldots, n\}} \zeta_{P_{B}}(t)^{(-1)^{\# B+1}}
$$

is rational. Q.E.D.

We shall now reduce the proof of Theorem 3 to 
LEMma 8. Let $U \subseteq k^{r}$ be definable, defined by an atomic formula in $L_{\tau^{\prime}, k}$ of type

$$
\varphi_{n}\left(p_{0}\left(x_{1}, \ldots, x_{r}\right), \ldots, p_{n}\left(x_{1}, \ldots, x_{r}\right)\right)
$$

with $p_{i}\left(x_{1}, \ldots, x_{r}\right) \in k\left[x_{1}, \ldots, x_{r}\right] \quad(i=1, \ldots, n)$ (obviously, we mean that $U$ is defined by a formula of $L_{\tau, k}$ equivalent to $\varphi_{n}\left(p_{0}(\bar{x}), \ldots, p_{n}(\bar{x})\right)$; then $\pi_{U}(t)$ is rational.

Before we prove Lemma 8, we shall reduce the proof of Theorem 3 to it, i.e., show that Theorem 3 follows from Lemmas 7 and 8 .

Let $U$ be a definable set; it has been proved in $\$ 3$ that $\Sigma^{\prime}$ admits elimination of quantifiers, hence we may assume $U$ defined by a quantifier-free formula $\varphi$ in the language $L_{\tau^{\prime}, k}$, i.e., $U$ is the union of sets defined by formulae of type

$$
\bigwedge_{i=1}^{\mu} p_{i}(\bar{x})=0 \wedge \bigwedge_{j=1}^{\nu} \varphi_{n_{j}}\left(p_{n_{j}, 0}(\bar{x}), \ldots, p_{n_{j}, n_{j}}(\bar{x})\right) \wedge \bigwedge_{k=1}^{\xi} q_{k}(\bar{x})
$$

$$
\neq 0 \wedge \bigwedge_{m=1}^{n} \neg \varphi_{n_{m}}\left(p_{n_{m}, 0}(\bar{x}), \ldots, p_{n_{m}, n_{m}}(\bar{x})\right) .
$$

Again, since intersections of sets defined by formulae of type (*) are again defined by formulae of type (*), it will suffice to prove that the $\zeta$-functions of sets defined by formulae of type (*) have the required property.

We are now reduced to sets $U$ defined by formulae of type (*). To proceed, we start by freeing ourselves from the restrictions imposed by the defining axiom for $\varphi_{m}$ in case we are interpreting this relation in a field with $m$ elements.

LemMa 9. Let $U$ be defined by a formula $\varphi$ of type (*). Let $\psi^{\prime}$ be obtained from $U$ by replacing each occurrence of $\varphi_{m}\left(p_{m, 0}(\bar{x}), \ldots, p_{m, m}(\bar{x})\right)$ by $\exists z\left(p_{m, 0}(\bar{x})+\cdots+p_{m, m}(\bar{x}) z^{m}=0\right)$. Let $U^{\prime}$ be the set defined by $\varphi^{\prime}$. Then, if $\pi_{U^{\prime}}(t)$ is rational, so is $\pi_{U}(t)$.

Proof. Let

$$
\begin{aligned}
& A=\left\{m \in \mathbf{Z}_{>0} \mid \varphi_{m} \text { occurs in } \varphi \text { and } m=q^{s}, \text { for some } s \in \mathbf{Z}_{>0}\right\}, \\
& B=\left\{s \in \mathbf{Z}_{>0} \mid q^{s}=m, \text { for some } m \in A\right\} .
\end{aligned}
$$

If $B=\varnothing, \nabla s \in \mathbf{Z}_{>0}, U_{s}=U_{s}^{\prime}$ hence $N_{s}(U)=N_{s}\left(U^{\prime}\right)$ and the result is obvious. But if $B \neq \varnothing$, it certainly is finite. Also, $\forall s \in \mathbf{Z}_{>0}, s \notin B \Rightarrow N_{s}(U)=N_{s}\left(U^{\prime}\right)$. Hence $\pi_{U}(t)=\Sigma_{s=1}^{\infty} N_{s}(U) t^{s}=\Sigma_{s=1}^{\infty} N_{s}\left(U^{\prime}\right) t^{s}-\Sigma_{s \in B} N_{s}\left(U^{\prime}\right) t^{s}+$ $\Sigma_{s \in B} N_{s}(U) t^{s}$. From the finiteness of $B$ and the rationality of $\Sigma_{s=1}^{\infty} N_{s}\left(U^{\prime}\right) t^{s}$ 
we immediately conclude the rationality of $\pi_{U}(t)$. Q.E.D.

So in everything that follows we may replace $\varphi_{m}\left(p_{m, 0}, \ldots, p_{m, m}\right)$ by $\exists z\left(p_{m, 0}+\cdots+p_{m, m^{2}} z^{m}=0\right)$.

As before, in formulae of type (*) we may assume $\xi \leqslant 1$ by replacing $\bigwedge_{k=1}^{\xi} q_{k}(\bar{x}) \neq 0$ by $\Pi_{k=1}^{\xi} q_{k}(\bar{x}) \neq 0$; similarly. We may assume $\eta \leqslant 1$; indeed:

$$
\begin{aligned}
& \Sigma \vdash \bigwedge_{m=1}^{n} \neg \exists z\left(p_{n_{m}, 0}(\bar{x})+\cdots+p_{n_{m}, n_{m}}(\bar{x}) z^{n_{m}}=0\right) \\
& \leftrightarrow \neg \exists z\left(\prod_{m=1}^{n}\left(p_{n_{m}, 0}(\bar{x})+\cdots+p_{n_{m}, n_{m}}(\bar{x}) z^{n_{m}}\right)=0 .\right.
\end{aligned}
$$

Furthermore, we can always assume $\xi=0$ :

$$
\begin{aligned}
& \Sigma \vdash q(\bar{x}) \neq 0 \wedge \neg \varphi_{n}\left(p_{0}(\bar{x}), \ldots, p_{n}(\bar{x})\right) \Leftrightarrow q(\bar{x}) \\
& \neq 0 \wedge \neg \exists z\left(p_{0}(\bar{x})+\cdots+p_{n}(\bar{x}) z^{n}=0\right), \\
& \Sigma \vdash q(\bar{x}) \neq 0 \wedge \neg \exists z\left(p_{0}(\bar{x})+\cdots+p_{n}(\bar{x}) z^{n}=0\right) \\
& \Leftrightarrow \neg \exists z\left(q(\bar{x})\left(p_{n}(\bar{x}) z^{n}+\cdots+p_{0}(\bar{x})\right)\right), \\
& \Sigma \vdash \neg \exists z\left(q(\bar{x})\left(p_{n}(\bar{x}) z^{n}+\cdots+p_{0}(\bar{x})\right)=0\right) \\
& \Leftrightarrow \neg \varphi_{n}\left(q(\bar{x}), \ldots, q(\bar{x}) p_{n}(\bar{x})\right) .
\end{aligned}
$$

Should $\eta=0$, we can always introduce the conjunct $7 \varphi_{1}(1.0)$. So, we may assume $\xi=0, \eta \leqslant 1$. We are now reduced to showing our result for sets defined by formulae of type

$$
\bigwedge_{i=1}^{\mu} p_{i}(\bar{x})=0 \wedge \bigwedge_{j=\mu+1}^{\nu} \varphi_{n_{j}}\left(p_{n_{j}, 0}(\bar{x}), \ldots, p_{n_{j}, n_{j}}(\bar{x})\right) .
$$

Indeed, if we get it for this case, then if we consider the set $U$ defined by $\bigwedge_{i=1}^{\mu} p_{i}(\bar{x})=0 \wedge \wedge_{j=1}^{\nu} \varphi_{n_{j}}(\cdots) \wedge \neg \varphi_{n}(\cdots)$, we observe that $U=V-$ $W$, where $V$ is defined by a formula of type (**) and $W$ by $\varphi_{n}(\cdots)$, so $N_{s}(U)$ $=N_{s}(V)-N_{s}(V \cap W)$, where $V \cap W$ is again defined by a formula of type (**).

Now to prove the result for a set $U$ defined by (**), it will suffice to establish the following:

Claim. Let $V_{i}$ be defined by $p_{i}(\bar{x})=0 \quad(i=1, \ldots, \mu)$ and by $\varphi_{n_{i}}\left(p_{n_{i}, 0}(\bar{x}), \ldots, p_{n_{i}, n_{i}}(\bar{x})\right)$ for $i=\mu+1, \ldots, \nu$. Then for all $B \subseteq\{1, \ldots$, v\}, $V_{B}=\bigcup_{i \in B} V_{i}$ is a set such that $d / d t \log \zeta_{V_{B}}(t)$ is rational.

Suppose we have proved the Claim: then 


$$
\begin{aligned}
N_{s}(U)=\#\left(\bigcap_{i=1}^{\nu}\left(V_{i}\right)_{s}\right) & =\sum_{B \subseteq\{1, \ldots, \nu\}}(-1)^{\# B} \#\left(V_{B}\right)_{s} \\
& =\sum_{B \subseteq\{1, \ldots, \nu\}}(-1)^{\# B} N_{s}\left(V_{B}\right) .
\end{aligned}
$$

Now to prove the Claim:

Let

$$
\begin{aligned}
& B_{1}=B \cap\{1, \ldots, \mu\}, \\
& B_{2}=B \cap \sum_{\{\mu+1, \ldots, \nu\}} V_{B}=\bigcup_{i \in B_{1}} V_{i} \cup \bigcup_{i \in B_{2}} V_{i}
\end{aligned}
$$

but $\bigcup_{i \in B_{1}} V_{i}$ can be defined by $\Pi_{i \in B_{1}} p_{i}(\bar{x})=0$, and $\bigcup_{j \in B_{2}} V_{j}$ can be defined by

$$
\exists z\left(\prod_{j \in B_{2}}\left(p_{n_{j}, n_{j}} z^{n_{j}}+\cdots+p_{n_{j}, 0}\right)=0\right),
$$

i.e., by $\varphi_{n}\left(q_{0}(\bar{x}), \ldots, q_{n}(\bar{x})\right)$, where $n=\Sigma_{j \in B_{2}} n_{j}$ and the $q_{i}(\bar{x})$ are adequately computed.

Hence $V_{B}$ is defined by

$$
\begin{gathered}
\prod_{i \in B_{1}} p_{i}(\bar{x})=0 \vee \varphi_{n}\left(q_{0}(\bar{x}), \ldots, q_{n}(\bar{x})\right) \text {, hence by } \\
\exists z\left(\pi p_{i}(\bar{x}) q_{n}(\bar{x}) z^{n}+\cdots+\pi p_{i}(\bar{x}) q_{0}(\bar{x})=0\right) \text {, hence by } \\
\varphi_{n}\left(\pi p_{i}(\bar{x}) q_{0}(\bar{x}), \ldots, \pi p_{i}(\bar{x}) q_{n}(\bar{x})\right),
\end{gathered}
$$

and the proof of Theorem 3 is actually reduced to Lemma 8.

Proof of Lemma 8. Let $U$ be defined by

$$
\varphi_{n}\left(p_{0}\left(x_{1}, \ldots, x_{r}\right), \ldots, p_{n}\left(x_{1}, \ldots, x_{r}\right)\right) ;
$$

by Lemma 9 we may assume $n>q$ :

$$
\begin{aligned}
U_{s}=\left\{\left(a_{1}, \ldots, a_{r}\right) \in k_{s}^{r} \mid \text { there exists } b \in k_{s}\right. \\
\text { such that } \left.p_{n}(\bar{a}) b^{n}+\cdots+p_{0}(\bar{a})=0\right\} .
\end{aligned}
$$

Let $f\left(x_{1}, \ldots, x_{r}, z\right)=p_{0}\left(x_{1}, \ldots, x_{r}\right)+\cdots+p_{n}\left(x_{1}, \ldots, x_{r}\right) z^{n} \in$ $k\left[x_{1}, \ldots, x_{r}, z\right]$. Let $V$ be the variety in $k^{r+1}$ defined by $f(\bar{x}, z)=0$ :

$$
V_{s}=\left\{(\bar{a}, b) \in k_{s}^{r+1} \mid f(\bar{a}, b)=0\right\} .
$$

Let 


$$
V_{s, i}=\left\{(\bar{a}, b) \in k_{s}^{r+1} \mid p_{n}(\bar{a}) z^{n}+\cdots+p_{0}(\bar{a}) \text { has } i\right. \text { distinct }
$$
roots in $k_{s}$ and $b$ is one of them\}

$(i=1, \ldots, n)$; obviously, we have $V_{s}=\bigcup_{i=1}^{n} V_{s, i}$ and we observe that

$$
N_{s}(U)=\# U_{s}=\sum_{i=1}^{n} \frac{\# V_{s, i}}{i} \text {. }
$$

Now let $H_{i}$ be the constructible $r+i$ set defined by

$$
f\left(\bar{x}, z_{1}\right)=0 \wedge \cdots \wedge f\left(\bar{x}, z_{i}\right)=0 \wedge \bigwedge_{\substack{k, m=1 \\ k \neq m}}^{i} z_{k}-z_{m} \neq 0 .
$$

By Lemma $6, \zeta_{H_{i}}(t)$ is rational. We also have $\left(H_{i}\right)_{s}=\left\{(\bar{a}, \bar{b}) \in k_{s}^{r+i} \mid f\left(\bar{a}, b_{k}\right)\right.$ $=0$ for $k=1, \ldots, i$ and $b_{k} \neq b_{m}$ if $k \neq m$ \}. Our aim is to compute $\# V_{s, i}$ from $N_{s}\left(H_{j}\right)$. For this purpose, let

$$
\begin{aligned}
E_{s, i} & =\left\{(\bar{a}, b) \in\left(H_{i}\right)_{s} \mid f(\bar{a}, z) \text { has exactly } i \text { distinct roots in } k_{s}\right\}, \\
F_{s, i} & =\left\{(\bar{a}, b) \in\left(H_{i}\right)_{s} \mid f(\bar{a}, z) \text { has }>i \text { distinct roots in } k_{s}\right\} .
\end{aligned}
$$

Of course, $\left(H_{i}\right)_{s}=E_{s, i} \stackrel{\circ}{\cup} F_{s, i}$ and also

$\#\left\{\bar{a} \in k_{s}^{r} \mid f(\bar{a}, z)\right.$ has exactly $i$ roots in $\left.k_{s}\right\}=\frac{1}{i !} \cdot \# E_{s, i}=\frac{\# V_{s, i}}{i}$,

hence $\# V_{s, i}=\# E_{s, i} /(i-1)$ !, and if we can compute $\# E_{s, i}=N_{s}\left(H_{i}\right)-\# F_{s, i}$ adequately, we are through.

Indeed, consider the map

$$
\begin{aligned}
\pi_{i}: \bigcup_{k=i+1}^{n} E_{s, k} & \rightarrow F_{s, i}, \\
\left(\bar{a}, b_{1}, \ldots, b_{i}, \ldots, b_{k}\right) & \rightarrow\left(\bar{a}, b_{1}, \ldots, b_{i}\right) .
\end{aligned}
$$

$\pi_{i}$ is certainly surjective and also

$$
k \neq k^{\prime} \Rightarrow \pi_{i}\left(E_{s, k}\right) \cap \pi_{i}\left(E_{s, k^{\prime}}\right)=\varnothing
$$

(indeed: $\left(\bar{a}, b_{1}, \ldots, b_{i}\right) \in \pi_{i}\left(E_{s, k}\right) \Rightarrow f(\bar{a}, z)$ has exactly $k$ roots). So

$$
\begin{aligned}
F_{s, i} & =\bigcup_{k=i+1}^{n} \pi_{i}\left(E_{s, k}\right), \text { hence } \\
\# F_{s, i} & =\sum_{k=i+1}^{n} \# \pi_{i}\left(E_{s, k}\right) .
\end{aligned}
$$

But for $k=i+1, \ldots, n, \# E_{s, k} /(k-i) !=\# \pi_{i}\left(E_{s, k}\right)$; hence \#E $E_{s, i}=N_{s}\left(H_{i}\right)-$ 
$\# F_{s, i}=N_{s}\left(H_{i}\right)-\Sigma_{j=i+1}^{n} \# E_{s, j} /(j-i)$ ! but we also know that $\# E_{s, n}=N_{s}\left(H_{n}\right)$ (from the definitions) and so we get

$$
\begin{aligned}
& \# V_{s, n}=\frac{1}{(n-1) !} N_{s}\left(H_{n}\right), \\
& \# V_{s, i}=\frac{1}{(i-1) !} \# E_{s, i}=\frac{1}{(i-1) !}\left(N_{s}\left(H_{i}\right)-\sum_{j=i+1}^{n}(j-1) ! \# V_{s, j}\right) \\
& \quad(i=1, \ldots, n-1) .
\end{aligned}
$$

This certainly determines each \# $V_{s, i}$ as a linear combination of the $N_{s}\left(H_{j}\right)$ $(j=1, \ldots, n)$ with rational coefficients (independent of $s)$; hence

$$
N_{s}(U)=\sum_{i=1}^{n} \frac{\# V_{s, i}}{i}
$$

is given by a linear combination of the $N_{s}\left(H_{j}\right)$ with rational coefficients, independent of $s$; hence the rationality of $\Sigma N_{s}(U) t^{s}$ follows from the rationality of $\Sigma N_{s}\left(H_{j}\right) t^{s}$. Q.E.D.

REMARK. The proof yields that $\pi_{U}(t)$ is rational for any definable set $U$. Certainly, $\zeta_{U}(t)$ may not be rational. However, this proof also shows that $\zeta_{U}(t)$ is always algebraic, indeed, it can always be written as the radical of a rational function.

5. Application. Let us consider the following:

DEFinition 10. Let $\theta: \widetilde{k}^{r} \rightarrow \widetilde{k}^{t}$ be a function; suppose we can find a $t$-tuple of polynomials $f_{1}, \ldots, f_{t} \in k\left[x_{1}, \ldots, x_{r}\right]$ such that for all $\left(a_{1}, \ldots\right.$, $\left.a_{r}\right) \in \tilde{k}^{r}, \theta\left(a_{1}, \ldots, a_{r}\right)=\left(f_{1}\left(a_{1}, \ldots, a_{r}\right), \ldots, f_{t}\left(a_{1}, \ldots, a_{r}\right)\right)$; then $\theta$ is called an $r$-t-morphism over $k$, and the $t$-tuple $\left(f_{1}, \ldots, f_{t}\right)$ is said to define $\theta$.

We can state the following

LEMMA 10. If $U$ is a definable r-set over $k$, and $\theta$ is an $r$ - t-morphism over $k$, then $\theta(U)$ is a definable $t$-set over $k$.

Proof. Say $U$ is defined by the formula $\varphi\left(x_{1}, \ldots, x_{r}\right)$ of $L_{\tau, k}$ and $\theta$ by the $t$-tuple $\left(f_{1}\left(x_{1}, \ldots, x_{r}\right), \ldots, f_{t}\left(x_{1}, \ldots, x_{r}\right)\right)$. Then it is trivial to check that $\theta(U)$ can be defined by the formula $\Psi\left(y_{1}, \ldots, y_{t}\right)$ given by

$$
\begin{aligned}
\exists x_{1} \cdots \exists x_{r}\left(y_{1}\right. & =f_{1}\left(x_{1}, \ldots, x_{r}\right) \wedge \cdots \wedge y_{t} \\
& \left.=f_{t}\left(x_{1}, \ldots, x_{r}\right) \wedge \varphi\left(x_{1}, \ldots, x_{r}\right)\right) . \quad \text { Q.E.D. }
\end{aligned}
$$

In particular, we get the following generalization of Dwork's result:

The logarithmic derivative of the zeta-function of the image of a variety by a morphism is rational. 


\section{REFERENCES}

1. James Ax, Solving diophantine problems modulo every prime, Ann. of Math. (2) 85 (1967), 161-183. MR 35 \#126.

2. - The elementary theory of finite fields, Ann. of Math. (2) 88 (1968), 239-271. MR 37 \#5187.

3. James Ax and S. Kochen, Diophantine problems over local fields. III. Decidable fields, Ann. of Math. (2) 83 (1966), 437-456. MR 34 \#1262.

4. J. L. Bell and A. B. Slomson, Models and ultraproducts. An introduction, NorthHolland, Amsterdam, 1969. MR 42 \#4381.

5. Z. I. Borevič and I. R. Šafarevič, Number theory, "Nauka", Moscow, 1964; English transl., Pure and Appl. Math., vol. 20, Academic Press, New York, 1966. MR 30 \#1080; 33 \#4001.

6. B. M. Dwork, On the rationality of the zeta function of an algebraic variety, Amer. J. Math. 82 (1960), 631-648. MR 25 \#3914.

7. G. E. Sacks, Saturated model theory, Math. Lecture Notes, Benjamin, New York, 1972.

8. J. R. Shoenfield, Mathematical logic, Addison-Wesley, Reading, Mass., 1967. MR 37 \#1224.

DEPARTMENT OF MATHEMATICS, UNIVERSITY OF CALIFORNIA, BERKELEY, CALIFORNIA 94720 